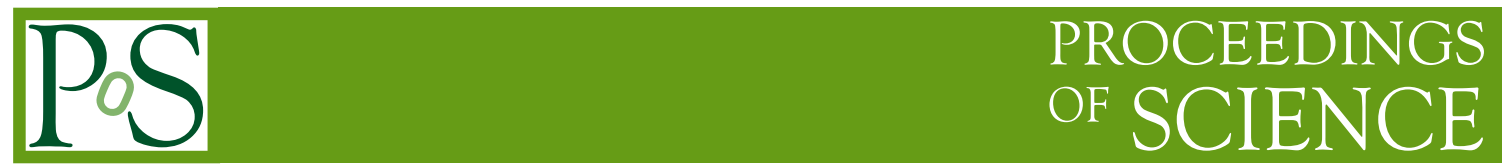

\title{
Results From Astroparticle Physics
}

Nathalie PALANQUE-DELABROUILLE*

CEA, IRFU, SPP, Centre de Saclay, F-91191 Gif sur Yvette Cedex, France

E-mail: nathalie@hep.saclay.cea.fr

\begin{abstract}
We present a general overview of recent results in cosmology and astroparticle physics. We first discuss the relevance of dark matter in the study of the optical, x-ray and weak-lensing data of the collision between two clusters of galaxies and how the alternative interpretation of Modified Newtonian Dynamics need to be adjusted to fit the observations. We present the current status of direct searches for WIMPs and mention the recent results of indirect searches. Turning to dark energy, we present two independent probes : distant type Ia supernovae, which gave the first evidence for dark energy, and baryon acoustic oscillations, which recently gave the most precise determination of the matter density in the universe from a single probe. Finally, we present a brief panorama of recent results in the field of high energy astroparticle physics.
\end{abstract}

Physics at LHC 2008

29 September - October 4, 2008

Split, Croatia

${ }^{*}$ Speaker. 
Astroparticle physics is a broad field encompassing topics related to particle physics, astronomy and cosmology. Using various complementary probes, it aims in particular at studying the composition and evolution of the universe as well as the most extreme natural phenomena.

Over the past 10 years, a wealth of new results has significantly improved our knowledge of the universe. Evidence coming from microwave background anisotropies (in particular with the results from the WMAP satellite [1]), from type Ia supernovae (first indication in 1998 from [2] and [3] and clear confirmation in 2006 with [4]), and from large scale structures (with the detection of the baryon acoustic oscillation in [5]) all imply that the Universe behaves as though it consists of $73 \pm 3 \%$ of dark energy, $23 \pm 3 \%$ of non baryonic dark matter, $4 \pm 0.4 \%$ baryonic dark matter and only $0.5 \%$ luminous matter (stars and galaxies). In addition, the total energy density of the Universe $\Omega_{\mathrm{T}}=1.01 \pm 0.01$ is consistent with that of a flat Universe. Sections 1 and 2 present some of the main evidence that led to this conclusion.

While the extent of the cosmic ray spectrum over 12 orders of magnitude in energy no longer surprises anyone, the cosmic accelerators that produce these particles are still not identified. In the past few years, the advent of new telescopes to study the high energy universe has brought some light to this long-standing enigma. These main results will be briefly presented in section 3 .

\section{Dark matter}

Since the original work of Zwicky in 1933 on the motion of galaxies in the gravitational field of the Coma cluster, evidence has accumulated indicating a significant amount of dark matter around galaxies and clusters of galaxies. One of the most convincing evidence for dark matter comes from the flat rotation curves of spiral galaxies. The main stream interpretation is to invoke an additional massive component called the "dark halo" whose mass can exceed by an order of magnitude that of the luminous component of the galaxy. Another possibility is to consider that Newtonian dynamics fails on the scale of galaxies when the gravitational field becomes too weak, imposing a modification of the laws of gravity as suggested by MOND theories (MOdified Newtonian Dynamics). One can then assume that the acceleration $a$ caused by a body of mass $M$ no longer goes as $G M / r^{2}$ at large radii $r$. Such a hypothesis is tested with the observation presented below.

\subsection{The "bullet" cluster}

The X-ray observation of any rich cluster of galaxies indicates that the galaxies are embedded in a large amount of gas. Its mass composition is typically $2-7 \%$ in galaxies, $10-30 \%$ in gas and $60-85 \%$ in dark matter. In such clusters, the baryon mass budget is therefore dominated by the gas. The density of galaxies is low enough that when two clusters collide, the galaxies can be considered collisionless and simply pass through the collision area. The gas, however, interacts electromagnetically and is significantly slowed down, thus remaining in the central region.

The mass distribution of the clusters after the collision is a good probe of their composition. In a MOND world, the mass is expected to be concentrated where most of the baryons are, i.e. with the gas in the collision area. In a WIMP world, the mass of the clusters is dominated by a collisionless non baryonic dark matter component expected to be located, after the collision, where the galaxies are. The mass distribution should then exhibit two distinct peaks corresponding to the mass centers of each cluster. Figure 1 shows the observations made on the bullet cluster [6]. The 
mass map follows the distribution of the galaxies, clearly indicating that a collisionless component makes up most of the mass of the clusters. MOND alone cannot explain this observation since mass is supposed to trace light, while non-baryonic dark matter in the form of WIMPs fits the picture perfectly. MOND, however, can still be saved by adding a contribution from 2-eV ordinary hot neutrinos [7] in an amount similar to that of the baryons in each cluster. A $2 \mathrm{eV}$ neutrino is within reach of the coming up KATRIN tritium beta decay experiment, and this solution should therefore be soon either confirmed or falsified.
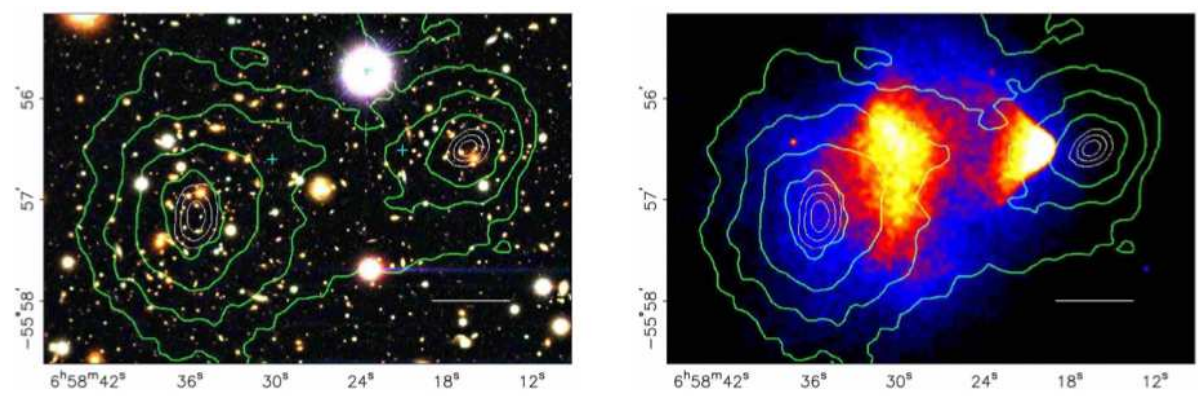

Figure 1: The bullet cluster in optical (left image) where the galaxies show, and in X-Ray (right image) where the hot gas shines. The green contours trace the mass distribution.

\subsection{WIMP searches}

WIMPs may be detected by their elastic scattering on detector nuclei. The detection challenge is considerable, since the signal is small because of the small recoil energy imparted by slow WIMPs, and the interaction rate is very low, always $\ll 1$ event/day $/ \mathrm{kg}$ of detector. Hence, backgrounds must be reduced by all available means: placing the detectors underground to filter out cosmic rays, using low-radioactivity materials in the detectors and in their immediate environment, and finally developing powerful event-by-event discrimination techniques in detecting and analyzing the signal.

Nuclear recoil signals typically will ionize the medium and release thermal energy (phonons) into it; in addition, scintillation light may be produced. Strong background discrimination can be achieved by detecting two of these signals, and requiring them to be consistent with a signal from nuclear recoil. This is the technique used by experiments like EDELWEISS (located in the Modane Lab, in the Fréjus tunnel) and CDMS (in the Soudan mine, USA) [8] with bolometers, but also by a recent generation of noble gas detectors such as XENON [9]. None of these experiments has claimed a DM detection and they have all published upper limits on the cross-section as a function of WIMP mass as illustrated in figure 2.

An annual signal modulation observed over 7 years - a possible WIMP signal due to the motion of Earth in an essentially stationary galactic particle distribution - has been observed by the DAMA/LIBRA experiment, which uses an array of $\mathrm{NaI}(\mathrm{Tl})$ scintillator crystals at the Laboratori Nazionali del Gran Sasso. This result, however, is put into question by the fact that it comes from a unique signature, scintillation light, and that the signal intensity is not compatible with upper limits from other experiments.

To probe the entire parameter space predicted by WIMP models will require over an order of magnitude improvement in sensitivity and thus the development of 1-ton detectors. Liquid detec- 


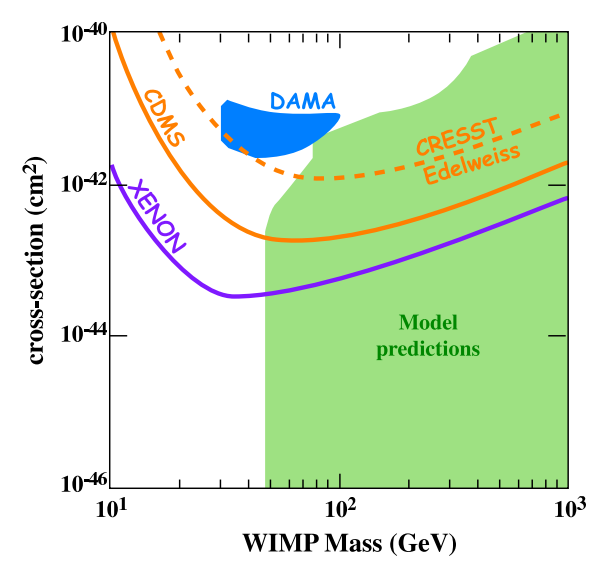

Figure 2: WIMP limits in the cross-section - mass plane compared to model predictions. The upper dashed line represents the current limits from CRESST and EDELWEISS; the upper continuous line is the CDMS limit; the lower continuous line is the latest limit from XENON with a $15 \mathrm{~kg}$ liquid xenon detector.

tors (whether xenon or argon) present the advantage over crystal detectors to be easily extendable to larger volumes and thus larger masses.

WIMPs can also be searched for indirectly by looking for their annihilation products (high energy photons with Integral or HESS, neutrinos with ANTARES or AMANDA) when WIMPs gather in dense regions of the galaxy or of the solar system. A very strong $511 \mathrm{keV}$ emission has been detected by the INTEGRAL satellite in an extended region surrounding the galactic center. An asymmetry in this emission was later discovered to be correlated with the distribution of XRay binaries in the plane of the galaxy, which could be responsible for up to $70 \%$ of the observed emission, leaving little space for a WIMP contribution [10]. A stable emission of $\mathrm{TeV}$ photons was observed by the HESS stereoscopic Cerenkov telescopes. Because the spectrum of the emission extends as a smooth power law up to $10 \mathrm{TeV}$, a WIMP interpretation of this signal would imply a particle with a mass beyond the $10 \mathrm{TeV}$ range, while the resulting spectrum would still be a poor fit to the data. A more plausible explanation lies in an astrophysical source such as the $3 \times 10^{6}$ solar mass black hole in the center of the galaxy, or the nearby Sgr A East supernova remnant [11].

\section{Dark Energy}

Certainly the most surprising cosmological result of the last decade is the discovery that the gravitational dynamics of the Universe is dominated by a "dark energy" whose density $\rho_{d e}$ is about twice that of matter and whose effective pressure $p_{d e}$ is negative. The present constraints lead to $p_{d e} / \rho_{d e} \equiv w \sim-1$. The case $w=-1$ corresponds to a cosmological constant $\Lambda=8 \pi G_{N} \rho_{d e}$ or equivalently to the dark energy being a simple vacuum energy. The negative pressure causes the expansion of the Universe to accelerate rather than decelerate as one would expect from the normal effect of the gravitational attraction of the matter in the Universe. The negative pressure also causes the density of dark energy to be relatively constant in time. The fact that the densities of matter and of radiation decrease as the Universe expands means that we live in a special epoch when the densities of matter and of dark energy are comparable. Quantitatively, the current densities of 
matter and dark energy in units of the critical density are $\Omega_{d e} \sim 0.73$ and $\Omega_{m} \sim 0.27$ giving a total density of $\Omega_{T} \sim 1$ corresponding to a flat Universe as favored by the WMAP results.

\subsection{Supernovae}

The first evidence for dark energy came from the photon flux from high redshift type Ia supernovae $[2,3]$. For a given redshift, the flux is lower in the case of an accelerating universe than for a decelerating universe. For supernovae at redshift of $z \sim 0.5$ the difference is about $40 \%$ between the case $\left(\Omega_{m}, \Omega_{d e}\right)=(0.27,0.73)$ and that for a critical matter dominated Universe $\left(\Omega_{m}, \Omega_{d e}\right)=(1,0)$. The precision of the supernovae measurements has recently improved with the publication of the first results from the Supernova Legacy Survey[12] on the Canada-France-Hawaii Telescope. The program benefits from a $1 \mathrm{deg}^{2} \mathrm{CCD}$ imager, large enough to monitor simultaneously of order ten visible supernovae with $z<1$. The relatively uniform temporal sampling of the curves in four pass bands is a great improvement over the original observations that generally discovered supernovae at their maximum light, giving precise measurements only on the falling side the the light curve.

If one assumes a flat universe, as favored by the spectrum of anisotropies of the Cosmic Background Radiation [1], then the SNLS results imply $\Omega_{d e}=1 .-\left(0.263 \pm 0.042_{\text {stat }} \pm 0.032_{\text {sys }}\right)$. Combined with the results of the Sloan Digital Sky Survey [5] discussed below, the SNLS results imply $w=1.023 \pm 0.090_{\text {stat }} \pm 0.054_{\text {sys }}$ for $\Omega_{T}=1$, indicating that the dark energy behaves very much like a cosmological constant.
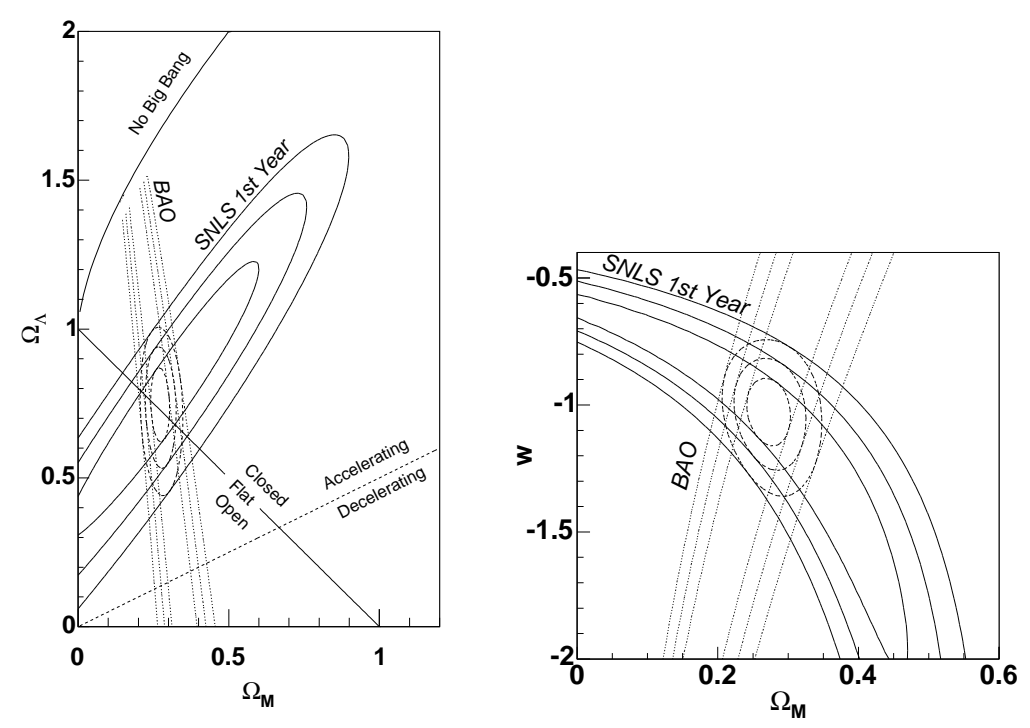

Figure 3: The constraints on $\Omega_{m}$ and $\Omega_{\Lambda}=\Omega_{d e}$ (left) and on $\Omega_{m}$ and $w$ (right) from the Supernova Legacy Survey (SNLS) and the BAO observations of the Sloan Digital Sky Survey (SDSS).

The Supernova evidence for dark energy is based on the fact that the flux from high redshift supernovae is lower than expected. Such an effect could also, in principle, be caused by intergalactic absorption. Another possibility is that supernovae in the distant past were simply dimmer than at present because of different astrophysical conditions. Because of these two caveats, it is important to have independent evidence for the existence of dark energy. Such evidence now exists with the observation of Baryon Acoustic Oscillations (BAO) by the SDSS [5]. 


\subsection{Baryon acoustic oscillations}

The SDSS is a project to obtain photometric and spectroscopic data on over 1 million galaxies over about $1 / 3$ of the sky and at redshifts less than $\sim 0.6$. Its galaxy correlation function shows a peak at $\sim 150 \mathrm{Mpc}$ [5]. This preferred distance is a prediction of models with Cold Dark Matter (CDM). In such models, structures form from density fluctuations in the primordial Universe. Consider a small positive fluctuation of matter at the origin. As the Universe evolves, the CDM component of the fluctuation will more or less stay in place since it consists of pressureless slow particles. On the other hand, the electrons, baryons and photons in the fluctuation are strongly coupled by Compton and Coulomb scattering and therefore form a fluid that supports sound waves. The photon-baryon-electron component of the fluctuation then spreads out from the original position in a spherical wave, stopping only at the time of electron-proton recombination when the pressure falls to zero. The original point fluctuation thus yields the original fluctuation at the origin surrounded by a shell at the distance a sound wave can travel between the big bang and recombination, the so-called sonic horizon at recombination. Later on when galaxies form, they will have a small preference for being separated by this distance. The sonic horizon thus gives a standard ruler that can be used in a manner similar to the way type Ia supernovae are used as standard candles.

To first approximation, the sonic horizon is proportional to $1 / \sqrt{\Omega_{m}}$. The peak seen in the SDSS correlation function then determines precisely the matter density in the universe [5]: $\Omega_{m}=$ $0.273 \pm 0.025$ assuming $w=-1$ and $\Omega_{T}=1$.

\section{High energy astroparticle}

While particles with energies as high as a few $10^{20} \mathrm{eV}$ have been detected for several decades, the cosmic accelerators responsible for the observed particles are still unknown.

A step forward has been made possible by the HESS experiment which uses 4 Cerenkov telescopes to detect the light emitted by the secondaries of cosmic rays as they propagate through the atmosphere. Although it is generally accepted that the only sources capable of supplying the energy required to accelerate the bulk of Galactic cosmic rays are supernova explosions, and even though the mechanism of particle acceleration in expanding supernova remnant (SNR) shocks is thought to be well understood theoretically, unequivocal evidence for the production of high-energy particles in supernova shells has proven remarkably hard to find. On several occasions [13, 14], the HESS experiment has detected the emission of $\mathrm{TeV}$ gamma-rays correlated to the known $\mathrm{X}$-ray emission from supernova remnants, thus demonstrating that very-high-energy particles are accelerated there. The energy spectrum indicates efficient acceleration of charged particles to energies beyond 100 $\mathrm{TeV}$, consistent with current ideas of particle acceleration in young SNR shocks.

A long-standing question concerns the existence or not of a GZK-cutoff at the high energy end of the cosmic ray spectrum due to the interaction of these ultra-high energy (UHE) particles with the cosmic microwave background. The AUGER experiment uses two independent techniques to measure the flux of UHE particles, thus allowing a cross-calibration of the energy determination by either technique : 4 fluorescence telescopes measure the ultra-violet light emitted from nitrogen excited by charged particles, and 1600 water tanks detect the electrons, photons and muons produced in the air showers initiated by the interaction of the UHE particle in the upper atmosphere. The 
AUGER collaboration reported a strong suppression of the spectrum above $5 \times 10^{19} \mathrm{eV}$, illustrated in figure 4, in agreement with the prediction by Greisen, Zatsepin and Kuz'min [15].

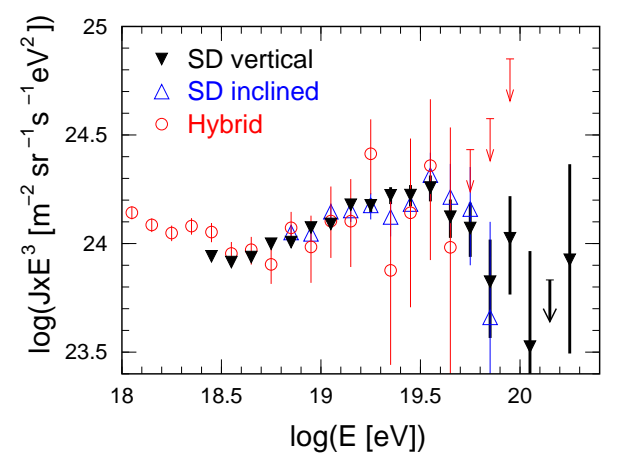

Figure 4: Energy spectrum from surface detectors (SD) only and from cross-calibration between surface detectors and fluorescence telescopes (hybrid).

Another remarkable result reported by the AUGER colaboration [16] is the observation of an anisotropy in the arrival directions of the UHE cosmic rays, which are correlated with the positions of relatively nearby active galactic nuclei. The correlation has maximum significance for cosmic rays with energy greater than $6 \times 10^{19} \mathrm{eV}$ and active galactic nuclei at a distance less than about $75 \mathrm{Mpc}$. The observed correlation is compatible with the hypothesis that cosmic rays with the highest energies originate from extra-galactic sources close enough so that their flux is not significantly attenuated by interaction with the cosmic background radiation.

\section{References}

[1] D. Spergel et al., ApJ Suppl. 170, 377 (2006).

[2] A. Riess et al., AJ 116, 1009 (1998).

[3] S. Perlmutter et al., ApJ 517, 565 (1999).

[4] P. Astier et al., A\&A 447, 31-48 (2006).

[5] D. Eisenstein et al., $A J$ 633, 560-574 (2005).

[6] D. Clowe et al., ApJ 648, L109-113 (2006).

[7] G. Angus et al., ApJ 654, L13-16 (2007).

[8] D.S. Akerib et al., Phys. Rev Lett, 96, 011302 (2006).

[9] J. Angle et al., Phys. Rev Lett, 100, 021303 (2007).

[10] G. Weidenspointer et al., Nature, 451, 159 (2008).

[11] F. Aharonian et al., Phys. Rev Lett, 97, 221102 (2006).

[12] P. Astier et al. Astron.Astrophys. 447 31-48 (2006).

[13] F. Aharonian et al., Nature, 432, 75 (2004).

[14] F. Aharonian et al., $A \& \&$ A, 437, L7 (2005).

[15] J. Abraham et al., Phys. Rev Lett, 101, 061101 (2008).

[16] J. Abraham et al., AP 29, 188 (2008). 